\title{
Parsing Economic Technology Matrices by Triangular Decomposition*
}

\author{
REINER WOLFF \\ Department of Quantitative Economics, University of Fribourg, Ave de Beauregard 13, \\ CH-1700 Fribourg, Switzerland; E-mail: Reiner.Wolff@unifr.ch
}

(accepted)

\begin{abstract}
An input-output technology with intermediate inputs is said to be productive or viable if it allows for positive net amounts of every commodity. Each division or sector will then contribute less than one unit of direct and indirect intermediate inputs to the making of one unit of its own output. Equivalently, the leading principal minors of the technology's associated Leontief matrix are all positive (Hawkins-Simon condition). We demonstrate that the minors can be checked efficiently in the course of a triangular decomposition of this matrix.
\end{abstract}

Key words:

JEL Classification number: C00, D57, O40

\section{Introduction}

Consider an input-output technology with linear activities where $n$ divisions or sectors $(n \geq 2)$ are linked by an $n \times n$ matrix $\mathbf{A}=\left(a_{i j}\right)$ of physical production coefficients for intermediate inputs. We assume that all matrix elements are nonnegative real numbers. Furthermore, denote as $\mathbf{I}$ the $n \times n$ identity matrix such that $\mathbf{B}:=\mathbf{I}-\mathbf{A}$ represents the technology's associated Leontief matrix. Then, how can we identify a technology which is productive or viable (cf. Dorfman et al. 1958, pp. 210-215)? Intuitively, each division or sector $i$ should contribute less than one unit of direct and indirect intermediate inputs to the making of one unit of its own output. (The term 'indirect' refers to commodities $i$ which are incorporated in inputs from other sectors $j$.) Hence, certain restrictions must be placed on $\mathbf{A}$ and B, respectively, in order to permit positive net outputs of every good, provided that there are enough primary resources.

A major literature result states that an input-output technology is productive or viable if and only if all leading principal minors $\operatorname{det}(\mathbf{B}(1: k, 1: k))$ of $\mathbf{B}$ are positive

* Earlier versions of this paper have been presented at the 1999 meeting of the Swiss Society of Economics and Statistics, at the OR 2002 joint conference of the Austrian, German and Swiss Societies of Operations Research as well as during seminars at the Universities of Fribourg, Karlsruhe and Potsdam. 
(e.g. Nikaido (1975, Chapters $1 \&$ 3), Takayama (1985, Chapter 4), Mas-Colell et al. (1995, pp. 155-157)). We will show that this so-called Hawkins-Simon condition (Hawkins/Simon (1949)) can be checked efficiently with a triangular decomposition or factorization algorithm.

\section{A Technology Parser}

Our technology parser benefits from the following result of advanced matrix algebra:

THEOREM. If $\mathbf{B}$ is a real-valued, regular $n \times n$ matrix and $\operatorname{det}(\mathbf{B}(1: k, 1: k)) \neq$ 0 for all $k=1, \ldots, n-1$, then there exists a unique pair of real-valued $n \times n$ matrices $\mathbf{L}=\left(l_{i j}\right)$ and $\mathbf{U}=\left(u_{i j}\right)$ which are lower and upper triangular, respectively, such that $\mathbf{B}=\mathbf{L} \mathbf{U}$, while $l_{i i}=1$ for all $i$.

Proof. See Golub/Van Loan (1996, pp. 97-98).

For the purpose of illustration, consider the following example of an inputoutput technology and associated Leontief matrix $\mathbf{B}$ for $n=3$ divisions or sectors:

$$
\begin{aligned}
& \underbrace{\left(\begin{array}{ccc}
0.30 & -0.20 & -0.20 \\
-0.06 & 0.84 & -0.46 \\
-0.09 & -0.10 & 0.56
\end{array}\right)}_{=\mathbf{B}}=\underbrace{\left(\begin{array}{ccc}
1 & 0 & 0 \\
l_{21} & 1 & 0 \\
l_{31} & l_{32} & 1
\end{array}\right)}_{=\mathbf{L}} \underbrace{\left(\begin{array}{ccc}
u_{11} & u_{12} & u_{13} \\
0 & u_{22} & u_{23} \\
0 & 0 & u_{33}
\end{array}\right)}_{=\mathbf{U}} \\
& =\left(\begin{array}{ccc}
u_{11} & u_{12} & l_{21} u_{13}+u_{23} \\
l_{21} u_{11} & l_{21} u_{12}+u_{22} \\
l_{31} u_{11} & l_{31} u_{12}+l_{32} u_{22} & l_{31} u_{13}+l_{32} u_{23}+u_{33}
\end{array}\right)
\end{aligned}
$$

Note that $\mathbf{B}$ is regular, as $\operatorname{det}(\mathbf{B})=0.096 \neq 0$. Also note that $\operatorname{det}(\mathbf{B}(1: 1,1: 1))=$ $0.30 \neq 0$ and $\operatorname{det}(\mathbf{B}(1: 2,1: 2))=0.24 \neq 0$. Hence, $\mathbf{L}$ and $\mathbf{U}$ exist and are unique. In particular, we can employ a straightforward procedure to solve (1) for the nine $\left(=n^{2}\right)$ unknown l's and $u$ 's. This procedure is known as the Crout LU algorithm. It operates along the main diagonal of $\mathbf{B}$ from the top-left to the bottom-right corner:

- Step 1: $u_{11}=0.30, u_{12}=-0.20, u_{13}=-0.20$,

$$
l_{21}=-0.06 / u_{11}=-0.20, l_{31}=-0.09 / u_{11}=-0.30 .
$$

- Step 2: $u_{22}=0.84-l_{21} u_{12}=0.80, u_{23}=-0.46-l_{21} u_{13}=-0.50$,

$$
l_{32}=\left(-0.10-l_{31} u_{12}\right) / u_{22}=-0.20 \text {. }
$$

- Step 3: $u_{33}=0.56-l_{31} u_{13}-l_{32} u_{23}=0.40$.

We thereby find that all elements of $\mathbf{B}$ are needed to calculate the entire set of unknowns. However, the subset $u_{11}, u_{12}, u_{22}$, and $l_{21}$ turns out to be independent 
of the entries to the third row and column of $\mathbf{B}$. This subset effectively comes from the LU decomposition of $\mathbf{B}(1: 2,1: 2)$. In the same way, $u_{11}$ also does not depend on the second row and column of $\mathbf{B}$ and constitutes the (trivial) LU decomposition of $\mathbf{B}(1: 1,1: 1)$.

Altogether, the factorization of a general $n \times n$ Leontief matrix B implicitly incorporates the factorization of all leading principal submatrices of $\mathbf{B}$ in one turn (the last submatrix being identical to $\mathbf{B}$ ). Consequently, since $\mathbf{L}$ and $\mathbf{U}$ are triangular:

$$
\begin{aligned}
\operatorname{det}(\mathbf{B}(1: k, 1: k)) & =\operatorname{det}(\mathbf{L}(1: k, 1: k)) \operatorname{det}(\mathbf{U}(1: k, 1: k)) \\
& =\prod_{i=1}^{k} l_{i i} \prod_{i=1}^{k} u_{i i} \\
& =\prod_{i=1}^{k} u_{i i} \quad \text { for all } k=1, \ldots, n .
\end{aligned}
$$

The leading principal minors $\operatorname{det}(\mathbf{B}(1: k, 1: k))$ of $\mathbf{B}$ can thus be checked one after another as the factorization of $\mathbf{B}$ proceeds: The first occurrence of a zero or negative coefficient $u_{k k}$ in step $k$ of Crout's algorithm

$$
\begin{array}{ll}
u_{k i}=b_{k i}-\sum_{j=1}^{k-1} l_{k j} u_{j i} & \text { for all } i=k, \ldots, n, \\
l_{i k}=\frac{1}{u_{k k}}\left(b_{i k}-\sum_{j=1}^{k-1} l_{i j} u_{j k}\right) & \text { for all } i=k+1, \ldots, n,
\end{array}
$$

would indicate that $\mathbf{B}$ represents a non-viable production plan. The factorization could then already be halted. Otherwise, it terminates conformably with all $u_{k k}>0$. At the same time, $u_{k i}, l_{i k} \leq 0$ for all $i>k$. In particular, once an element $b_{i j}$ of B has been evaluated for its associated $l$-value or $u$-value, this element will never be used again. As a consequence, the factorization of $\mathbf{B}$ can be done in situ, e.g., replacing the above $3 \times 3$ Leontief matrix by

$$
\left(\begin{array}{lll}
u_{11} & u_{12} & u_{13} \\
l_{21} & u_{22} & u_{23} \\
l_{31} & l_{32} & u_{33}
\end{array}\right)=\left(\begin{array}{ccc}
0.30 & -0.20 & -0.20 \\
-0.20 & 0.80 & -0.50 \\
-0.30 & -0.20 & 0.40
\end{array}\right) \text {. }
$$

We suggest to perform pivot search as a measure to reduce roundoff. Partial pivoting (row interchanges) will, in general, do. Consequently, if $\mathbf{P}$ stands for an appropriate $n \times n$ permutation matrix, we operate on $\mathbf{P B}$ rather than on $\mathbf{B}$. An algorithm, which checks the leading principal minors of $\mathbf{B}$ has to be modified accordingly: 
LEMMA. If the LU decomposition of the Leontief matrix $\mathbf{B}$ of a viable or productive technology is implemented with partial pivoting (row interchanges), then each pivot choice $b_{i k}<0(i>k)$ will result in a negative diagonal element $u_{k k}$ of $\mathbf{U}$.

Proof. Suppose that in the course of the factorization we swap two successive matrix rows $k$ and $i=k+1$ of $\mathbf{B}$. Let $\mathbf{P}$ stand for the corresponding permutation matrix. Note that we actually swap the same rows of the triangular matrix $\mathbf{L}$ (as $\mathbf{P B}=\mathbf{P L U})$ such that the minor $\left(\begin{array}{cc}c_{k+1, k} & 1 \\ 1\end{array}\right)$ will be generated along the diagonal positions $k$ and $k+1$ of the new matrix PL. Hence, the $k$ th leading principal minor of this matrix equals $l_{k+1, k}$ and will be strictly negative due to a pivot choice $b_{k+1, k}<0$. The final $n-k$ leading principal minors of PL come out as -1 . This means that the last $n-k+1$ leading principal minors of $\mathbf{P L}$, and thus of $\mathbf{P B}$, assume a negative value. Consequently, the factorization of $\mathbf{P B}$ (instead of $\mathbf{B}$ ) must result in a negative diagonal element $u_{k k}$ of $\mathbf{U}$ while all subsequent diagonal elements of $\mathbf{U}$ maintain their positive sign. Finally, observe that any division or sector $i>k+1$ can be re-labeled to become division or sector $k+1$ beforehand.

Table I provides an adaptation of a standard version of Crout's algorithm in pseudo code (e.g. Press et al. (1987, pp. 33-36)). The algorithm completes the first column of $\mathbf{L}$, then turns to the second row of $\mathbf{U}$, and so on. At the same time, the entries to the respective lower-right part of $\mathbf{B}$ are updated. On output, $\mathbf{B}$ will contain the LU decomposition of (a row-wise permutation of) the corresponding Leontief matrix according to (4), provided that the factorization was successful. If not, then the Boolean variable fail will return 'true' $(=-1)$ which signifies that a zero or negative coefficient $u_{k k}$ was encountered prior to a pivot search and that the factorization of $\mathbf{B}$ had been halted.

Table I. Technology Parser (in situ).

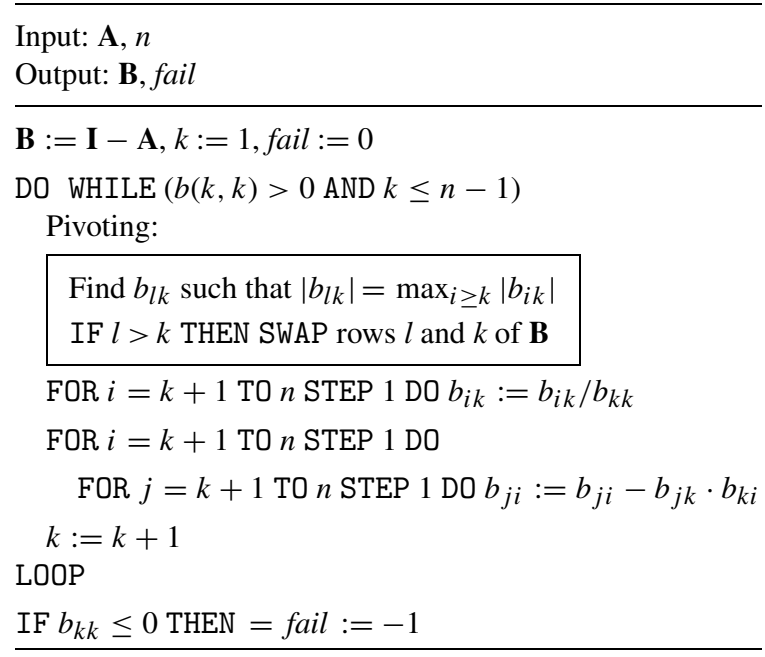


Hence, in case of a non-productive technology, the algorithm may terminate before the decomposition is complete, and thereby reduce its run time.

\section{Concluding Remarks}

In our experiments, it took less than one second on a Pentium $4 \mathrm{CPU}(2.40 \mathrm{GHz})$ to identify a viable technology for dense (hypothetical) production systems of up to 200 divisions or sectors and an associated double-precision matrix B. The computational burden became more noticeable for larger systems, as Crout's method has an $O\left(n^{3}\right)$ count of approximately $2 n^{3} / 3$ floating-point operations. These results suggest that Table I provides an effective workhorse method for parsing economic technology matrices of reasonable practical sizes.

The literature offers further characterizations of a productive or viable technology. They can be useful when the numerical stability of a parsing algorithm is a critical issue and indirect methods are called for, as in cases of very large technology matrices or matrices that are ill conditioned. While these methods may be computationally more demanding, it can still be shown that a technology is always productive if $\|\mathbf{A}\|<1$ holds for an arbitrary matrix norm like, e.g., the row-sum or column-sum norms (Brauer-Solow row-sum or column-sum conditions). There are thus sufficient conditions for the viability of a production system which can be checked first with little extra effort. The interested reader is referred to Takayama (1985, Chapter 4).

\section{References}

Dorfman, R., Samuelson, P.A. and Solow, R.M. (1958). Linear Programming and Economic Analysis, MacGraw-Hill, New York.

Golub, G.H. and Van Loan, C.F. (1996). Matrix Computations, 3rd ed., Johns Hopkins University Press, Baltimore.

Hawkins, D. and Simon, H.A. (1949). Note: Some conditions of macroeconomic stability. Econometrica, 17, 245-248.

Nikaido, H. (1975). Introduction to Sets and Mappings in Modern Economics, 3rd ptg., North Holland, Amsterdam.

Mas-Colell, A., Whinston, M.D. and Green, J.R. (1995). Microeconomic Theory, Oxford University Press, New York.

Press, W.H., Flannery, B.P., Teukolsky, S.A. and Vetterling, W.T. (1987). Numerical Recipes: The Art of Scientific Computing, 5th ptg., Cambridge University Press, Cambridge.

Takayama, A. (1985). Mathematical Economics, 2nd ed., Cambridge University Press, Cambridge. 